Scientific Paper

\title{
Interpretation of Gamma Index for Quality Assurance of Simultaneously Integrated Boost (SIB) IMRT Plans for Head and Neck Carcinoma
}

\author{
Maria ATIQ ${ }^{1}$, Atia ATIQ ${ }^{1, a}$, Khalid IQBAL ${ }^{2}$, Quratul ain SHAMSI ${ }^{1}$, Farah ANDLEEB ${ }^{1}$, Saeed Ahmad BuZdAR ${ }^{1}$ \\ ${ }^{I}$ Department of Physics, The Islamia University of Bahawalpur \\ ${ }^{2}$ Shaukat Khanum Memorial cancer hospital \& research center Lahore Pakistan \\ ${ }^{a}$ E-mail address: atiaatiq@hotmail.com
}

(received 14 June 2017; revised 17 September 2017; accepted 3 October 2017)

\begin{abstract}
Objective: The Gamma Index is prerequisite to estimate point-by-point difference between measured and calculated dose distribution in terms of both Distance to Agreement (DTA) and Dose Difference (DD). This study aims to inquire what percentage of pixels passing a certain criteria assure a good quality plan and suggest gamma index as efficient mechanism for dose verification of Simultaneous Integrated Boost Intensity Modulated Radiotherapy plans.

Method: In this study, dose was calculated for 14 head and neck patients and IMRT Quality Assurance was performed with portal dosimetry using the Eclipse treatment planning system. Eclipse software has a Gamma analysis function to compare measured and calculated dose distribution. Plans of this study were deemed acceptable when passing rate was 95\% using tolerance for Distance to agreement (DTA) as 3mm and Dose Difference (DD) as 5\%.

Result and Conclusion: Thirteen cases pass tolerance criteria of 95\% set by our institution. Confidence Limit for DD is 9.3\% and for gamma criteria our local CL came out to be $2.0 \%$ (i.e., $98.0 \%$ passing). Lack of correlation was found between DD and $\gamma$ passing rate with $\mathrm{R}^{2}$ of 0.0509 . Our findings underline the importance of gamma analysis method to predict the quality of dose calculation. Passing rate of $95 \%$ is achieved in $93 \%$ of cases which is adequate level of accuracy for analyzed plans thus assuring the robustness of SIB IMRT treatment technique. This study can be extended to investigate gamma criteria of $5 \% / 3 \mathrm{~mm}$ for different tumor localities and to explore confidence limit on target volumes of small extent and simple geometry.
\end{abstract}

Key words: simultaneous integrated boost SIB; distance to agreement DTA; dose difference DD; portal dosimetry; quality assurance QA; head and neck.

\section{Introduction}

Intensity Modulated Radiation Therapy (IMRT) is an attractive technique that provides highly precise dose around the target volume for the treatment of head and neck cancer [1]. To selectively increase dose per fraction to the target site Simultaneous Integrated Boost (SIB), also known as dose painting technique, is used [2]. Mohan et al [4] introduced the concept of SIB IMRT: Advantageous technique in terms of better conformity to target, shorter treatment time, better dose hotspot control and reduced exposure of radiations to Organs at Risk (OARs) such as parotids, spinal cords, optic nerve, optic chaisma, brain stem, and oral cavity [3-4]. This technique is specifically found useful for head and neck carcinoma due to histologically heterogeneous nature of tumor and low dose radiation tolerance to normal surrounding tissues [5-6]. To achieve local control of disease, high dose of radiation in the range of $70 \mathrm{~Gy}$ is required in the treatment of head and neck cancer [1]. This complex treatment process demands high level of quality assurance in treatment delivery. Quality Assurance (QA) consist of comparing delivered dose distribution of IMRT plans to phantom with two dimensional dose distribution by Treatment Planning System (TPS). The dose is verified using Thermoluminescent Detectors (TLDs), films or polymeric gels.

Gamma Index is an essential tool to ensure accuracy of applied plans and its potential to detect drawbacks in intended planar dose distribution [7]. Since the introduction of Gamma Index by Low et al, it has been adopted for IMRT QA by various groups [8-10]. Pass fail decision for the evaluation of gamma index was proposed by Depuydt et al [9]. IMRT specific QA guidelines are given in European SocieTy of Radiotherapy and Oncology ESTRO [11] and American Association of Physicists in Medicine AAPM Task Group TG 119 report [12]. For comparison between measured and calculated dose distribution, gamma evaluation method is adopted in this study, which quantifies both absolute Dose Difference and Distance to Agreement criteria [11]. DTA is a distance between reference point and closest data point in the compared dose distribution that manifests the same dose [9]. DTA measure works well only in high dose gradient regions. So composite analysis of DTA and DD is made to work in both 
high and low dose gradient regions. The test passes only when both DD and DTA criteria pass. This index is formulated such that when its value is $\leq 1$ the patient plan is accepted and when $\gamma$ value is greater than one, plan is rejected [13-14]. Thus gamma score is a measure of goodness of treatment plan which presents percentage of dose points that satisfy acceptance criteria [15].

This study aims to assure quality of SIB IMRT plans using $\gamma$ analysis software to compare measured and calculated dose distribution and to determine what percentage of points passes a certain criteria.

\section{Material and Method}

In this study fourteen $H \& N$ cancer patients were treated with SIB IMRT technique. Each patient underwent Computed Tomography (CT) simulation, acquired with slice thickness of $3 \mathrm{~mm}$. Two target volumes PTV1 and PTV2 were obtained with prescription doses of $70 \mathrm{~Gy}$ (at the rate of $2 \mathrm{~Gy}$ in 35 fractions) and 55.4 Gy respectively. All SIB IMRT plans included seven fields and gantry angle was fixed at $0^{\circ}, 51^{\circ}$, $102^{\circ}, 153^{\circ}, 204^{\circ}$, and $255^{\circ}$ using $6 \mathrm{MV}$ photon beam delivered by DHX Clinac equipped with 120 leaf Multileaf Collimator MLCs. The study was conducted at Shaukat Khanum Memorial Cancer Hospital \& Research Center Lahore and dosimetric information for the patients was obtained using Electronic Portal Imaging Devices (EPIDs). Initially EPIDs were used for patient position verification, however their use has been subsequently extended to secure dosimetric information for field verification before treatment [16].

Calculated and measured fluence maps were compared using portal dosimetry analysis module incorporated in Eclipse software. Eclipse software has a Gamma analysis function which compare two images and pixels. ARIA 11 Eclipse treatment planning and Varian Clinac ${ }^{\circledR}$ DHX; equipped with EPID which consist of Amorphous Silicon (aSi) detector technology, aSi active detector area is $30 \times 40 \mathrm{~cm}$, resolution is $512 \times 384$ pixels $(0.78 \mathrm{~mm})$, maximum imager resolution is $1024 \times 768(0.39 \mathrm{~mm})$. AAPM TG 119 recommend the DD/DTA passing criteria of $90 \%$ for $3 \% / 3 \mathrm{~mm}$ and ESTRO study proposed criteria of $4 \% / 3 \mathrm{~mm}$ with $95 \%$ passing criteria [11-12]. Many pilot studies suggested different criteria for DD/DTA parameter [17-18], however recommendations and reports for interpretation of gamma passing rate are scarce until now and these criteria remain empirically determined [17]. International Commission of Radiation Units and Measurements ICRU recommend less stringent $\gamma$ score of $5 \% / 5 \mathrm{~mm}$ criteria [19], however there is no general consensus. Plans of this study were deemed acceptable when passing rate was $95 \%$ using tolerance for DTA as $3 \mathrm{~mm}$ and DD as $5 \%$. Local confidence limit was explored using mean percentage of gamma passing points and SD. In present study Lower Limit of Agreement (LLA) and Upper Limit of Agreement (ULA) for dose difference were calculated in terms of Count Units (CU) [20].
$L L A=\mid$ Average $\mid-2 \times S D$

Eq. 1

$U L A=\mid$ Average $\mid+2 \times S D$

Eq. 2

Confidence Limits (CL) for Gamma analysis were found using the equations:

$L L A=\mid 100-$ Average $\mid-2 \times S D$
$U L A=\mid 100-$ Average $\mid+2 \times S D$ Eq. 3

Table 1 shows numerical value of tolerance level for average gamma. IMRT delivery technique and equipment, data analysis software and quality assurance policy in hospital are few factors that influence the acceptance criteria $[7,11]$.

Table 1. Evaluation filter for acceptability of $\gamma$ evaluations for verification of IMRT plans [21-11].

\begin{tabular}{ccc}
\hline \hline & Range & Appraisal and Approach \\
\hline \multirow{2}{*}{ Average $\gamma$} & $0-0.5$ & Acceptable. \\
& $0.5-0.6$ & Need further evaluation \\
& $>0.6$ & Not Acceptable \\
\hline \multirow{2}{*}{ Maximum $\gamma$} & $<1.5$ & Acceptable. \\
& $1.5-2.0$ & Need further evaluation \\
\hline \hline
\end{tabular}

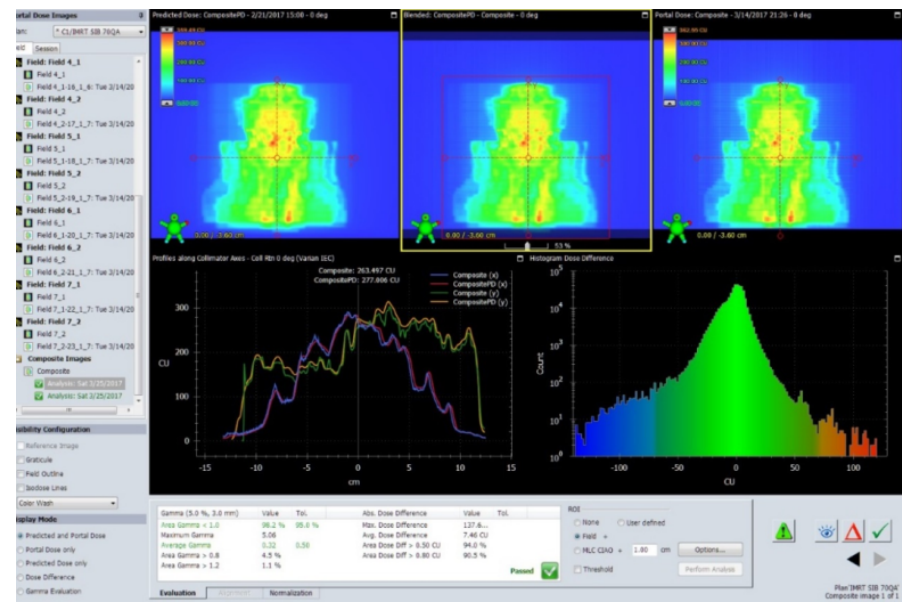

Figure 1. Pictorial representation of predicted and measured dose on left and right with composite dose in the center of upper row. Gamma histogram can be seen on right side of the second row. Evaluation table at the bottom row shows gamma evaluation results.

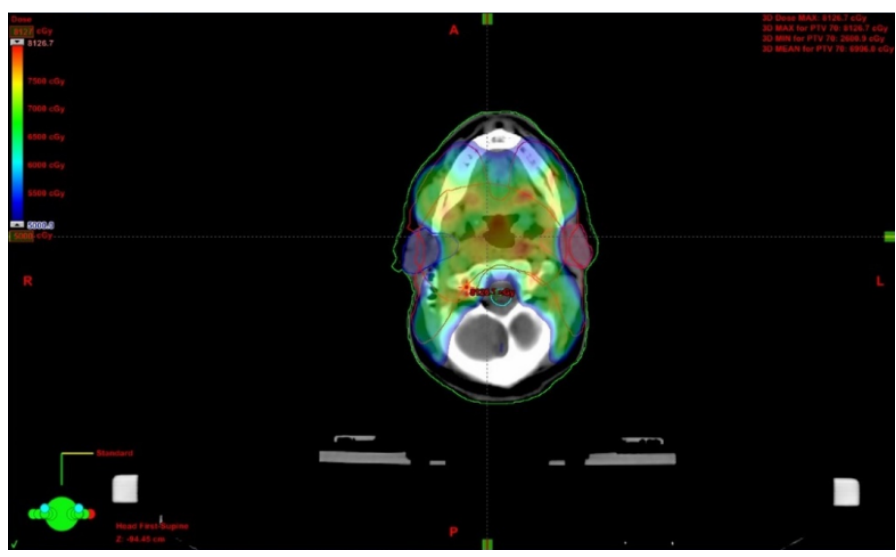

Figure 2. Representation of 7 field treatment plan of head and neck patient with $6 \mathrm{MV}$ measured with portal dosimetry. 


\section{Results}

Gamma Evaluation software generates gamma parameters such as area gamma <1, maximum gamma, average gamma, area gamma $>0.8$ and area gamma $>1.2$. Two of these scalar parameters i.e average $\gamma$ and maximum $\gamma$, which is 99th percentile of gamma distribution, evaluated by 2D gamma evaluation method for comparison of EPID reconstructed and planned dose distribution were investigated in this study.

Table 2 depicts portal dosimetry results for 14 SIB IMRT cases. Maximum and average gamma values of this study were $2.66 \pm 2.38$ and $0.304 \pm 0.07$ respectively for $5 \% / 3 \mathrm{~mm}$ criteria. In present study a higher value of $5 \% / 3 \mathrm{~mm}$ was used for plan evaluation. Table 3 shows mean $\gamma$ pass rate of $98 \% \pm 0.018$. To verify quality of IMRT plans ULA and LLA and confidence limits, that is based on normal distribution, of gamma index and DD were also determined. The confidence limits for dose difference for treatment site were measured by using formula $\mid$ mean|+1.96SD. Likewise confidence limit of Percentage of points passing gamma score of $5 \% / 3 \mathrm{~mm}$, was calculated using |100-mean|+1.96SD [22]. Confidence limit for DD is $9.3 \%$ and for gamma criteria our local CL came out to be $2.0 \%$ (i.e., $98.0 \%$ passing). Instead of factor 1.96 multiplying factor "2" was used to calculate LLA and ULA.

Figure 3 displays a composite analysis plan indicating pass/fail criteria for gamma index which was composite of both DTA and DD. Thirteen out of fourteen cases were above the line that deemed to automatically pass tolerance criteria of $95 \%$ set by our institution; those below line must be reviewed by a medical physicist to decide if the plan was acceptable for treatment or not.

Percentage dose difference related dose calculated with EPID to the dose delivered by DHX Clinac expressed in calibration unit CU. The dose difference criteria used throughout this paper always refers to the percentage of the maximum field dose. Graphical representation of percentage dose difference and gamma score is represented in Figure 5.

\section{Discussion}

Deconstruction of SIB IMRT plan for quality assurance requires complex method such as $\gamma$ function. Lower values of gamma parameters, Avg $\gamma$ and $\operatorname{Max} \gamma$, indicate better agreement between predicted and measure dose. Values of $\gamma$ area $>1$ and average $\gamma<1$ are restricted to high dose gradient regions. High gamma values are confined to low dose gradients as suggested by literature [23]. Zijtveld et al. reported values of average gamma to be $0.43 \pm 0.13$ for 75 treatment cases, which are in agreement with the results of present study [24]. Results of parameter maximum gamma of present study do not fall within acceptable range as demonstrated in Table 1. Higher gamma values may be due to small difference in dose where dose was not reformed by MLCs as most likely occurred in the present study. Howell et al. suggest gamma parameters to be higher for head and neck carcinoma, which may be due to leakage of dose into regions of low dose-gradient [23].
Table 2. Dosimetric results of $14 \mathrm{H} \& N$ patients for SIB IMRT technique.

\begin{tabular}{ccccccc}
\hline \hline \multirow{2}{*}{ Treatment Site } & \multicolumn{2}{c}{ Max Gamma } & & \multicolumn{2}{c}{ Average Gamma } \\
\cline { 2 - 3 } \cline { 5 - 6 } & Mean & 1 SD & & Mean & 1 SD \\
\hline Head And Neck & 2.66 & 2.38 & & 0.304 & 0.07 \\
\hline \hline
\end{tabular}

Table 3. Statistical analysis of Dose Difference and percentage of pixels passing $\gamma$ criteria of $5 \%$ of $3 \mathrm{~mm}$ with associated confidence limit.

\begin{tabular}{ccc}
\hline \hline & Mean & -0.0151 \\
Dose & SD & 0.04 \\
Difference & ULA & 0.095 \\
& LLA & -0.0648 \\
& CL & 0.093 \\
\hline & Mean & 98 \\
$\boldsymbol{\gamma} \mathbf{c r i t e r i a}$ & SD & 0.018 \\
$\mathbf{5 \% / 3 m m}$ & ULA & 2.036 \\
& LLA & 1.96 \\
& CL & 2.036 \\
\hline \hline
\end{tabular}

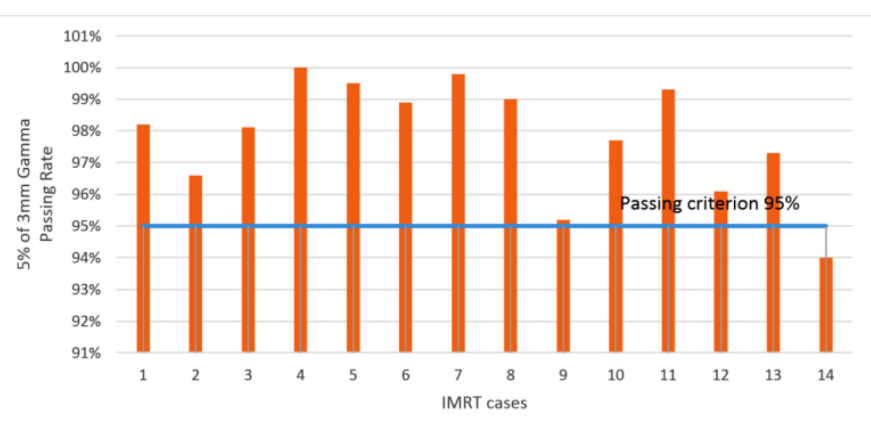

Figure 3. Gamma Results of 14 IMRT cases of Head and neck cancer for SIB IMRT technique

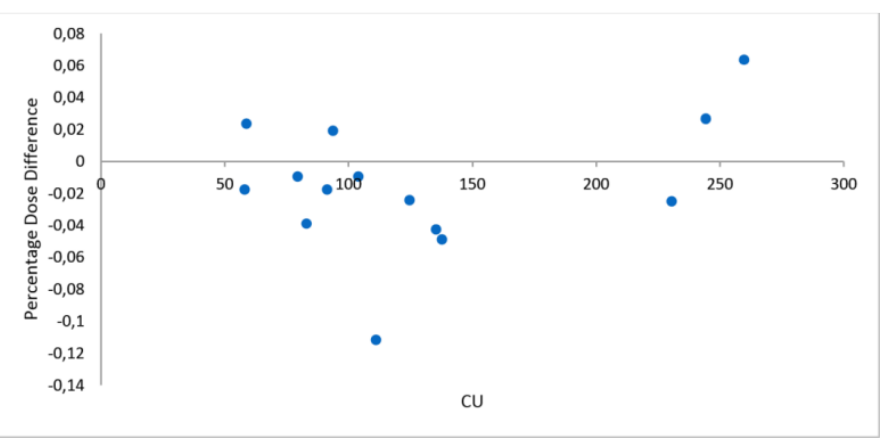

Figure 4. Plot of Percentage Dose Difference between calculated and measured dose as a function of control units $\mathrm{CU}$.

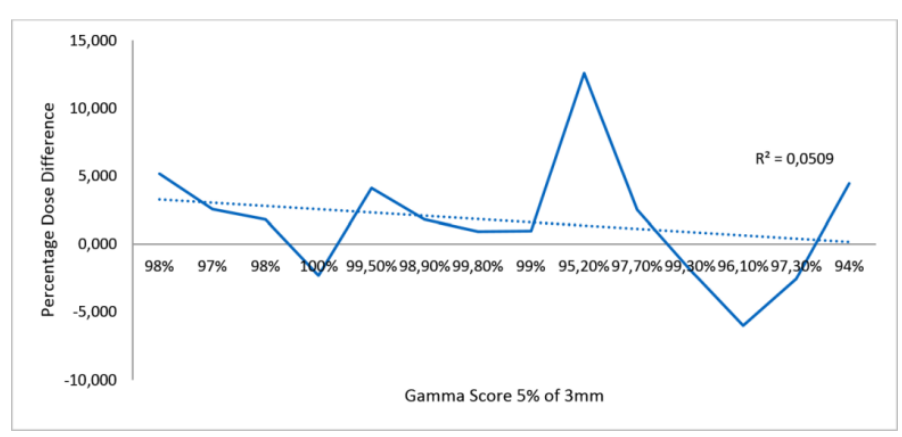

Figure 5. Gamma passing rate vs. percentage dose difference for 13 patients. 
Recently Childress et al performed IMRT verification using 850 films and concluded $5 \% / 3 \mathrm{~mm}$ as preferred acceptance criteria for gamma index which is also followed in this study [25]. This criteria is also recommended in AAPM TG 53 [26]. Mean gamma pass rate came out to be $98 \%$. Confidence limits suggest a reasonable standard for IMRT delivery. Confidence limits of this study for gamma criteria were near enough to the guidelines AAPM TG 119, hence performance of TPS in our institution is verified [12]. Our overall local confidence limit for gamma was determined to be $2.04 \%$, which was better than the value of reference $2(10 \%)$ using criteria of $3 \% / 3 \mathrm{~mm}$ [22]. There is slight deviation of our results from AAPM guidelines for dose difference with CL determined to be $9.3 \%$. Literature on international recommendations report that planning and dose delivery in radiotherapy can never be perfect [22]. Precision of dose calculation algorithm has large impact on dose difference. Hence confidence limit for DD depends on the proper assessment of dose calculation. [20]. Previous studies suggest that positioning error of multileaf collimator, inadequate dosimetric data of MLC in treatment planning system, complexity of tumor site, mode of delivery, and mishandling of dosimeter by user may cause discrepancy in measurement of dose difference [27-30]. Difference in results were due to fact that liberal gamma criteria of $5 \% / 3 \mathrm{~mm}$ was used in this study instead of more stringent criteria of 3\%/3 $\mathrm{mm}$ as adopted in AAPM TG 119.

The poor gamma scores of one of the patients, as indicated in Figure 3, were due to the field edges being clipped [2]. The failed data point lie predominately in high dose-gradient region [22]. One out of fourteen plans failed gamma evaluation test due to inevitable uncertainties in dosimetric measurements. Literature suggest that certain failure rate have to be ignored [31]. Acceptable values for $\gamma$ index for head and neck cancer are consistent with the values previously reported [1]. Lower gamma scores can attribute to complexity of tumor site $[1,12]$.
IMRT Gamma analysis results permits the clinicians to predict the effect of delivered dose on patient's anatomy. Although $\gamma$ index is very advantageous in determining the quality of plan, yet it only display number of data points without giving information about their spatial site [15]. In Figure 4, percentage dose difference of most of $H \& N$ plans were negative which suggest under dosage, same results reported by Chung et al [22]. Figure 5 demonstrates that there is a lack of correlation between DD and $\gamma$ passing rates, same results reported in literature [20], with $\mathrm{R}^{2}$ of 0.0509 .

\section{Conclusion}

In this study gamma criteria of $5 \% / 3 \mathrm{~mm}$, recommended in AAPM TG-53, for portal dosimeter based IMRT QA of fourteen head and neck patients was investigated. Our findings underline the importance of gamma analysis method to predict the quality of dose calculation. Passing rate of $95 \%$ is achieved in $93 \%$ of cases which is adequate level of accuracy for analyzed plans thus assuring the robustness of SIB IMRT treatment technique. Our local confidence limits for dose difference and gamma criteria suggest a reasonable standard for IMRT delivery. Confidence limits of this study for gamma criteria were near enough to the AAPM TG 119 guidelines; hence, performance of TPS in our institution is verified. However there is slight deviation of our results for dose difference from AAPM guidelines with CL determined to be 9.3\%. International recommendations suggest that planning and dose delivery in radiotherapy can never be perfect. Thus portal dosimetry is considered as efficient way for verifying quality of SIB IMRT treatment. This study can be extended to investigate gamma criteria of $5 \% / 3 \mathrm{~mm}$ for different tumor localities and to explore confidence limit on target volumes of small extent and simple geometry.

\section{References}

[1] Kouloulias V, Antypas C, Liakouli Z, et al. The first implementation of IMRT technique for head \& neck and prostate cancer patients in public sector in Greece: feasibility, treatment planning and dose delivery verification using the delta (4PT) Pre-Treatment volumetric quality assurance system. J BUON. 2014;20(1):196-205.

[2] Roxby KJ, Crosbie JC. Pre-treatment verification of intensity modulated radiation therapy plans using a commercial electronic portal dosimetry system. Australas Phys Eng Sci Med. 2010;33(1):51-57.

[3] Franceschini D, Paiar F, Meattini I, et al. Simultaneous integrated boost-intensity-modulated radiotherapy in head and neck cancer. Laryngoscope. 2013;123(12):E97-103.

[4] Mohan R, Wu Q, Manning M, Schmidt-Ullrich R. Radiobiological considerations in the design of fractionation strategies for intensity-modulated radiation therapy of head and neck cancers. Int J Radiat Oncol Biol Phys. 2000;46(3):619-630.

[5] Krishnan J, Rao S, Hegde S, Shetty J. A Dosimetric Comparison of Double Arc Volumetric Modulated Arc Therapy with Large Field Intensity Modulated Radiation Therapy for Head and Neck Cancer. IJMPCERO. 2015;4(4):353-363.

[6] Suzuki M, Nakamatsu K, Kanamori S, et al. Feasibility study of the simultaneous integrated boost (SIB) method for malignant gliomas using intensity-modulated radiotherapy (IMRT). Jpn J Clin Oncol. 2003;33(6):271-277.

[7] Elawady RA, Attalla EM, Elshemey WM, et al. Dose verification of intensity modulated radiotherapy in head and neck tumors. Int J Cancer Ther Oncol. 2014;2(3):02037. 
[8] Agazaryan N, Solberg TD, DeMarco JJ. Patient specific quality assurance for the delivery of intensity modulated radiotherapy. J Appl Clin Med Phys. 2003;4(1):40-50.

[9] Depuydt T, Van Esch A, Huyskens DP. A quantitative evaluation of IMRT dose distributions: refinement and clinical assessment of the gamma evaluation. Radiother Oncol. 2002;62(3):309-319.

[10] Low DA, Dempsey JF. Evaluation of the gamma dose distribution comparison method. Med Phys. 2003;30(9):2455-2464.

[11] Mijheer B, Georg D. Guidelines for the verification of IMRT. Brussels, Belgium: ESTRO; 2008.

[12] Ezzell GA, Burmeister JW, Dogan N, et al. IMRT commissioning: multiple institution planning and dosimetry comparisons, a report from AAPM Task Group 119. Med Phys. 2009;36(11):5359-5373.

[13] Tonigan JR. Evaluation of intensity modulated radiation therapy (IMRT) delivery error due to IMRT treatment plan complexity and improperly matched dosimetry data. MSc Thesis. The University of Texas. Houston, Texas; 2011.

[14] Varatharaj C, Ravikumar M, Sathiyan S, et al. Dosimetric verification of brain and head and neck intensity-modulated radiation therapy treatment using EDR2 films and 2D ion chamber array matrix. J Cancer Res Ther. 2010;6(2):179-184.

[15] Stasi M, Bresciani S, Miranti A, et al. Pretreatment patient-specific IMRT quality assurance: A correlation study between gamma index and patient clinical dose volume histogram. Med Phys. 2012;39(12):7626-7634.

[16] Caivano R, Califano G, Fiorentino A, et al. Clinically relevant quality assurance for intensity modulated radiotherapy plans: gamma maps and DVH-based evaluation. Cancer Invest. 2014;32(3):85-91.

[17] Li H, Dong L, Zhang L, et al. Toward a better understanding of the gamma index: Investigation of parameters with a surface-based distance method. Med Phys. 2011;38(12):6730-6741.

[18] Molineu A, Followill DS, Balter PA, et al. Design and implementation of an anthropomorphic quality assurance phantom for intensity-modulated radiation therapy for the Radiation Therapy Oncology Group. Int J Radiat Oncol Biol Phys. 2005;63(2):577-583.

[19] Grégoire V, Mackie TR. State of the art on dose prescription, reporting and recording in Intensity-Modulated Radiation Therapy (ICRU report No. 83). Cancer Radiother. 2011;15(6-7):555-559.

[20] Chaikh A, Desgranges C, Balosso J. Statistical methods to evaluate the correlation between measured and calculated dose using quality assurance method in IMRT. Int J Cancer Ther Oncol. 2015;3(4):

[21] Stock M, Kroupa B, Georg D. Interpretation and evaluation of the $\gamma$ index and the $\gamma$ index angle for the verification of IMRT hybrid plans. Phys Med Biol. 2005;50(3):399-411.

[22] Chung JB, Kim JS, Ha SW, Ye SJ. Statistical analysis of IMRT dosimetry quality assurance measurements for local delivery guideline. Radiat Oncol. 2011;6(1):27.

[23] Howell RM, Smith IP, Jarrio CS. Establishing action levels for EPID-based QA for IMRT. J Appl Clin Med Phys.2008;9(3):16-25.

[24] van Zijtveld M, Dirkx ML, de Boer HC, Heijmen BJ. Dosimetric pre-treatment verification of IMRT using an EPID; clinical experience. Radiother Oncol. 2006;81(2):168-175.

[25] Childress NL, White RA, Bloch C, et al. Retrospective analysis of 2D patient-specific IMRT verifications. Med Phys. 2005;32(4):838-850.

[26] Fraass B, Doppke K, Hunt M, et al. American Association of Physicists in Medicine Radiation Therapy Committee Task Group 53: quality assurance for clinical radiotherapy treatment planning. Med Phys. 1998;25(10):1773-1829.

[27] Jang SY, Liu HH, Mohan R. Underestimation of low-dose radiation in treatment planning of intensity-modulated radiotherapy. Int J Radiat Oncol Biol Phys. 2008;71(5):1537-1546.

[28] Deng J, Pawlicki T, Chen Y, et al. The MLC tongue-and-groove effect on IMRT dose distributions. Phys Med Biol. 2001;46(4):1039-1060

[29] Li JS, Lin T, Chen L, et al. Uncertainties in IMRT dosimetry. Med Phys. 2010;37(6):2491-2500.

[30] Das IJ, Ding GX, Ahnesjö A. Small fields: nonequilibrium radiation dosimetry. Med Phys. 2008;35(1):206-215.

[31] Ceberg C. A note on the interpretation of the gamma evaluation index. Journal of Physics: Conference Series. 2013;(444)1:012082. 